\title{
Filogeografía de Holothuria (Halodeima) inornata Semper, 1868 (Echinodermata: Holothuroidea)
}

\author{
Phylogeography of Holothuria (Halodeima) inornata Semper, 1868 (Echinodermata: Holothuroidea)
}

Elba Prieto-Rios ${ }^{1,2}$, Francisco Alonso Solís-Marín ${ }^{3}$, Giomar Helena Borrero-Pérez ${ }^{4}$ y Píndaro Díaz-Jaimes ${ }^{5}$

1 Facultad de Ciencias Biológicas, Biología, Universidad Nacional Mayor de San Marcos, Mesa de partes. Ciudad Universitaria de San Marcos, Av. Venezuela s/n Lima 1, Perú.

2 Posgrado en Ciencias del Mar y Limnología, Instituto de Ciencias del Mar y Limnología (ICML), Universidad Nacional Autónoma de México (UNAM), Apdo. Post. 70-305, 04510, México, D. F. México.

3 Colección Nacional de Equinodermos "Ma. E. Caso Muñoz". Laboratorio de Sistemática y Ecología de Equinodermos, ICML, UNAM.

4 Museo de Historia Natural Marina de Colombia (MHNMC). Instituto de Investigaciones Marinas y Costeras-INVEMAR “José Benito Vives De Andréis". Cerro Punta de Betín, Zona Portuaria, Santa Marta. Apartado aéreo 1016 y 873-Santa Marta, Colombia.

5 Laboratorio de Genética de Organismos Acuáticos. ICML, UNAM, Circuito interior s/n, Ciudad Universitaria, México D.F. México.

Email Elba Prieto-Rios: perprieto@hotmail.com

Email Francisco Solís-Marín: fasolis@cmarl.unam.mx

Email Giomar Borrero-Pérez:

Giomar borrero@invemar.org.co

Email Pindaro-Díaz-Jaimes: pindaro@cmarl.unam.mx

Citación:

Prieto-Rios E., F. Solís-Marín, G.H. BorreroPérez \& P. Díaz-Jaimes. 2014. Phylogeography of Holothuria (Halodeima) inornata Semper, 1868 (Echinodermata: Holothuroidea). Revista peruana de biología 21(2): 155 - 162 (Octubre 2014). doi: http://dx.doi.org/10.15381/rpb.v21i2.9818

Fuentes de flnanclamlento:

Proyecto a cargo de Alfredo Laguarda Figueras del LSEE, ICML No 204.

CONACYT-México, beca para estudios a Elba Prieto Rios, registro 447486

Proyecto a cargo de Píndaro Díaz Jaimes, Laboratorio de Genética de Organismos Acuáticos, ICML N 611

\section{Información sobre los autores:}

EPR, Desarrollo y ejecución de la investigación. FASM, Asesoramiento como especialista en equinodermos en la identificación y biología de $\mathrm{H}$. inornata. GHBP Asesoramiento y realización de los análisis de datos. PDJ, Asesoramiento en la metodología en laboratorio.

Presentado: 16/03/2014

Aceptado: $\quad 11 / 07 / 2014$

Publicado online: 07/10/2014

\section{Resumen}

Se evaluó la estructura genética de las poblaciones de Holothuria (Halodeima) inornata Semper, 1868, y se investigó cuáles podrían ser las barreras para el flujo de genes y procesos históricos. Se recolectaron muestras tratando de abarcar su ámbito de distribución, desde México hasta el norte de Perú. Con base en secuencias del gen COI se detectaron 118 haplotipos en 220 individuos y las diferencias entre dichos haplotipos fueron debidas a 97 sitios variables (21.41\%) en los 453 pb secuenciados. Se observó una alta diversidad de haplotipos $(\mathrm{h}=0.979)$ y moderada diversidad nucleotídica $(\pi=0.017)$. Para analizar la diferenciación genética, se utilizaron los valores de $\mathrm{F}_{\mathrm{s}}$, el test exacto de diferenciación poblacional y los análisis de varianza molecular (AMOVA). Estos análisis sugieren que existen dos poblaciones: las del norte, frente a las costas de: Sinaloa, Jalisco, Michoacán, Guerrero y Oaxaca; y las del sur, frente a las costas de: Chiapas, El Salvador, Panamá y Perú. Los acontecimientos históricos y los patrones oceanográficos podrían ser los principales factores que determinaron la dispersión y estructura de la población de $\mathrm{H}$. inornata, es probable que la población original se haya extendido inicialmente en el sur y luego hacia el norte. Además, la separación entre estas dos poblaciones podría deberse al Golfo de Tehuantepec, el cual está constituido por una serie de eventos tectónicos y oceanográficos que constituyen una barrera para el asentamiento de $H$. inornata.

Palabras clave: Equinodermos; Holothuria (Halodeima) inornata; ADNmt; Pacífico Oriental Tropical; Perú.

\section{Abstract}

Genetic structure of the populations of $H$. inornata was evaluated and the barriers for genetic flux and historic processes were investigated. Samples were collected trying to cover the distribution range of the species, from Mexico to northern Perú. Based on COI sequences, 118 haplotypes from 220 specimens were detected; the differences between such haplotypes were due to 97 variable sites $(21.41 \%)$ of the 453 bp sequenced. A high haplotype diversity $(h=0.979)$ and a moderate nucleotidic diversity were observed. The values of $F_{s t}$, the exact test of population differentiation, and the molecular variance analysis (AMOVA) were used in order to analyze the genetic differentiation. These analyses suggest the existence of two populations: northern, off the coasts of Sinaloa, Jalisco, Michoacán, Guerrero, and Oaxaca, and southern, off the coasts of Chiapas, El Salvador, Panamá and Perú. Historic events and oceanographic patterns may be the main factors determining dispersion and structure of $\mathrm{H}$ populations. It seems probable that the original population have extended first in the south and then northern. Besides, the split between these two populations may be due to several tectonic and oceanographic events constituting a barrier for $\mathrm{H}$. inornata settling.

Keywords: Echinoderms; sea cucumber; mtDNA; Eastern Tropical Pacific; Peru. 


\section{Introducción}

La región del Pacifico Oriental Tropical (POT) comprende aproximadamente entre los $25^{\circ} \mathrm{N}$ y los $4^{\circ} \mathrm{S}$. Dentro de esta región se han reconocido varias provincias zoogeográficas algunas basadas en el análisis de la distribución geográfica de peces costeros e invertebrados marinos (Hasting 2000, Robertson \& Cramer 2009, Briggs \& Bowen 2012), otras en la distribución de peces e invertebrados marinos actuales y fósiles presentes en la Formación Canoa (Landini et al. 2002) y en el porcentaje de endemismo de algunas especies de peces e invertebrados (Briggs 1974). Hasting (2000) propuso la Provincia Mexicana (desde Sinaloa-Mazatlán hasta Oaxaca), un Central American Gap (desde el Golfo de Tehuantepec hasta el Golfo de Fonseca), y la Provincia Panámica (desde el Golfo de Fonseca hasta el norte de Perú). Landini et al. (2002) reconocieron la Provincia Panámica (desde Mazatlán hasta el norte de Perú) y dentro de ella dos unidades distintas: la Mexicana $(\mathrm{m})$ que contiene las localidades de Sinaloa, Jalisco, Michoacán, Guerrero y Oaxaca, y la Panámica sensu stricto (p) que contiene las localidades de Chiapas, El Salvador, Panamá y Perú. Por último Briggs (1974) consideró la Provincia Mexicana (desde Sinaloa hasta El Salvador) y la Provincia Panámica (desde Nicaragua hasta del norte de Perú).

Muchas especies de peces e invertebrados tienen larvas que pueden ser capaces de viajar cientos de kilómetros durante su desarrollo, generalmente estas especies exhiben una estructura genética homogénea (panmíctica) en toda su área geográfica (Kinlan \& Gaines 2003); sin embargo, estructuras oceanográficas (v.g. corrientes, surgencias, eddies, jets) pueden determinar quiebres biogeográficos y patrones filogeográficos (Tellier et al. 2009, Zakas et al 2009, Brante et al 2012); por otro lado, el comportamiento de las larvas y su ecología, así como los efectos del paleoclima, también pueden generar o mantener patrones de la estructura genética que se desvíen de lo esperado (Sotka et al. 2004).

Un factor importante para la dispersión de larvas son las corrientes marinas. Para la región del POT, el cierre de la comunicación entre el Pacífico Tropical y el Caribe ocurrido hace 2.8 m.a., resultó un evento que condujo a los cambios en la circulación marina hasta llegar al patrón de circulación como actualmente se conoce (Coates et al. 2005, O’Dea et al. 2007). En el POT, la Corriente de California, que transcurre de norte a sur, es estacional y afecta solo las capas superficiales (Wyrtki 1965, Ruiz 2013); la Corriente Costera de Costa Rica es la rama más pronunciada de la circulación frente a Centroamérica y se mueve hacia el Noroeste y el Oeste llegando, una rama de la corriente, a ingresar en el Golfo de Tehuantepec (Quesada-Alpízar $\&$ Cortés 2006). A uno y otro lado de la zona ecuatorial están las corrientes denominadas Norecuatorial y Surecuatorial, que transportan agua superficial hacia el oeste (Kessler 2006). Por último, la circulación frente a la costa de Perú se destaca por la Corriente Peruana o Corriente de Humboldt que fluye hacia el noroeste y contribuye con sus aguas a la Corriente Surecuatorial como parte de la circulación (Allauca 1990, Morón 2000).

Si bien los sistemas de corrientes globales influyen sobre el transporte de las larvas, a menor escala existen otros factores que los modifican o influyen, como las surgencias, remolinos (eddies) y la fisiografía de la costa (Roughgarden et al. 1988, Nielsen \& Navarrete 2004, Kelly \& Palumbi 2010). En el norte de la región del Pacífico Oriental Tropical, se encuentra el
Golfo de Tehuantepec que ha merecido varios estudios porque en esa zona suceden condiciones de vientos que influencian las estructuras oceanográficas a meso escala, como corrientes, eddies y surgencias que afectarían la Corriente Costera de Costa Rica (Stumpf 1975, Alvarez et al. 1989, Fiedler 2002, Barton et al. 2009). Estas características pueden hacer de la zona del Golfo de Tehuantepec una potencial barrera para el flujo de genes de especies marinas, considerando que en su litoral se presentan lagunas y ríos, los cuales acarrean del continente sedimentos arenosos (de la Lanza 1991) lo cual limitaría el asentamiento de Holothuria inornata, la cual vive sobre fondos rocosos.

Holohturia inornata se distribuye en el Pacífico desde el Golfo de California hasta el norte de Perú; y en las Islas Clarión y Socorro (México), Isla del Coco (Costa Rica), Islas Galápagos (Ecuador) (Solís-Marín et al. 2009), Isla Foca e islas Lobos de Afuera (Perú). Estos organismos pueden alcanzar hasta $40 \mathrm{~cm}$ de longitud y en general habitan en aguas someras, entre 0 a $18 \mathrm{~m}$ de profundidad, en hábitats rocoso-arenosos (Alvarado \& Solís-Marín 2013). Esta especie es un recurso pesquero poco conocido; a pesar de su abundancia y accesibilidad en el litoral de México (Solís-Marín, datos no publicados). Se pesca artesanalmente y se explota ilegalmente en las Islas Galápagos, El Salvador y México, donde se ha reportado como severamente sobre explotada. En El Salvador, se encuentra en la lista de especies en peligro (FAO 2012).

La reproducción en $H$. inornata puede ser de forma asexual y sexual. En la población de H. inornata de Michoacán (México), la talla de maduración gonádica fue a los $22 \mathrm{~cm}$ de longitud, la edad calculada para este tamaño fue de 5 años (Ramos-Ramírez 2013). El ciclo reproductivo es semicontinuo; los periodos de reproducción abarcaron los meses de setiembre y octubre. La fecundación es externa, se desarrolla primero una larva auricularia y posteriormente una larva doliolaria, finalmente se establecen en el fondo del mar y pasan ahí su vida como adultos (RamosRamírez 2013).

Estudios genéticos de poblaciones utilizando diversos marcadores han sido realizados en equinodermos en distintos océanos. Para algunas especies de holoturias se han realizado trabajos en regiones del Océano Pacífico e Índico y el Atlántico oriental y el Mediterráneo (Uthicke et al. 1998, Arndt y Smith 1998, Uthicke y Benzie 2000, Uthicke y Benzie 2003, Chang et al. 2009, Borrero-Pérez et al. 2011). Sin embargo, no se han registrado estudios en la región del POT entre México y Perú, para ninguna especie de pepino de mar.

En este contexto, el presente trabajo investiga la estructura genética de $H$. inornata en la región del Pacifico Oriental Tropical, entre México y Perú. Los resultados indican que Holothuria inornata presenta una diversidad genética alta y una estructura genética diferenciada entre las unidades Mexicana y Panámica propuestas dentro de la provincia Panámica.

\section{Material y métodos}

Muestreo.- Se recolectaron muestras de $H$. inornata de nueve localidades tratando de abarcar toda su área de distribución geográfica. De norte a sur las muestras se obtuvieron frente a las costas de México: Sinaloa (playa Cerritos, $23^{\circ} 15^{\prime} \mathrm{N}$ ), Jalisco (Isla Cocinas, $\left.19^{\circ} 33^{\prime} \mathrm{N}\right)$, Michoacán (Caleta de Campos, $18^{\circ} 04^{\prime} \mathrm{N}$ ), Guerrero (Punta Maldonado, 16²19'N), Oaxaca (Puerto Ángel, $\left.15^{\circ} 39^{\prime} \mathrm{N}\right)$, Chiapas (Puerto Madero, $\left.14^{\circ} 42^{\prime} \mathrm{N}\right)$; costas de El 


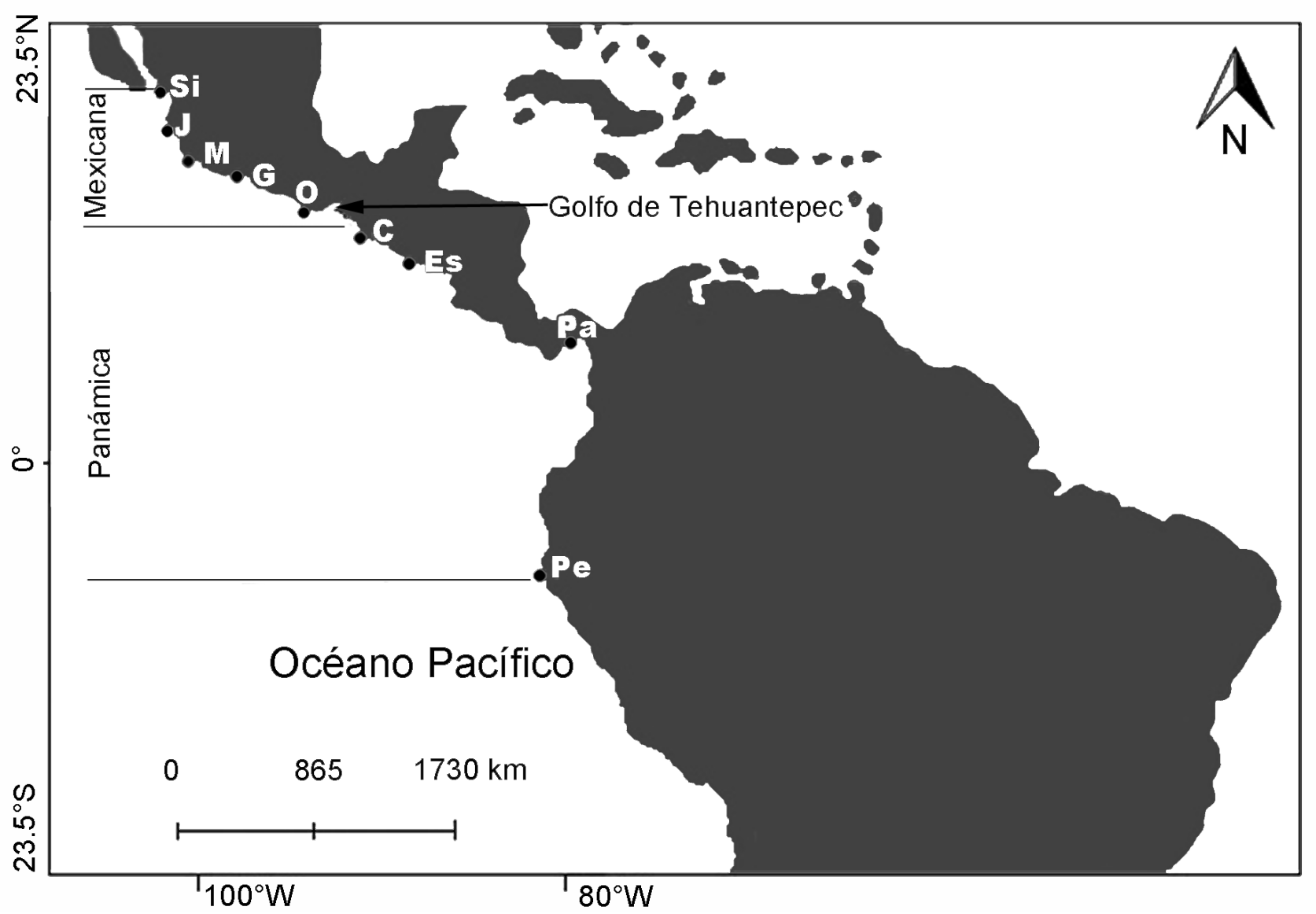

Figura 1. Mapa de los sitios muestreados para Holothuria (Halodeima) inornata. Si, Sinaloa; J, Jalisco; M, Michoacán; G, Guerrero; O, Oaxaca; C, Chiapas; ES, El Salvador; Pa, Panamá; Pe, Perú. Unidades dentro de la Provincia Panámica: unidad Mexicana y Panámica sensu stricto (Landini et al. 2002).

Salvador: Sonsonate (Los Cobanos, $\left.13^{\circ} 31^{\prime} \mathrm{N}\right)$, Panamá: San Carlos $\left(8^{\circ} 29^{\prime} \mathrm{N}\right)$ y Perú: Piura (Isla Foca, 5¹2’S) (Fig. 1). Los individuos fueron recolectados manualmente por buceo y se les extrajo un pedazo de tejido (pies ambulacrales) los que fueron guardados en etanol absoluto para el análisis molecular.

Extracción, amplificación y secuenciación.- El ADN genómico fue extraído según el procedimiento de Honey-Escandón et al. (2012), con algunas modificaciones. Para la limpieza de ADN se utilizó el QIAquick PCR Purification Kit (QIAGEN $\left.{ }^{\circledR}\right)$. Se amplificó un fragmento del gen mitocondrial Citocromo Oxidasa 1 (COI) utilizando iniciadores específicos diseñados para este estudio a partir de una secuencia de $H$. inornata de la página del GenBank, los iniciadores utilizados son: COI Forward 5'ATGGCTTTTCCCCGAATGAA3' y COI Reverse 5'ACACCATTCCTAGATACCCGA3'. Las amplificaciones se realizaron mediante la reacción en cadena de la polimerasa (PCRs) utilizando las mismas condiciones que Honey-Escandón et al. (2012). El ADN amplificado mediante las PCRs fue secuenciado, con un oligonucleótido, en el High Throughput Genomics Center, de la University of Washington, Department of Genome Sciences, EE.UU.

Diversidad genética.- Las secuencias se revisaron manualmente, y se editaron y alinearon con el programa BioEdit ver. 7.0.9.0 (http://www.mbio.ncsu.edu/bioedit/bioedit.html). La diversidad haplotípica $(\mathrm{H})$ y nucleotídica $(\pi)$, así como el número de sitios polimórficos (S), número promedio de diferencias nucleotídicas $(\mathrm{k})$ se obtuvieron por medio del programa ARLEQUIN 3.1 (http://cmpg.unibe.ch/softwarelarlequin35/) (Excoffier \& Lischer 2010).

Diferenciación poblacional.- Para entender la diferenciación o similitud de las poblaciones se utilizaron los estadísticos F de Wright (Wright 1951) calculados mediante la realización de 10100 permutaciones en ARLEQUIN. Para corroborar lo encontrado en los estadísticos $\mathrm{F}_{\mathrm{st}}$ se realizó el test exacto de diferenciación poblacional (longitud de la cadena de Markov, 100000 pasos) (Raymond \& Rousset 1995). Con el fin de evaluar el aislamiento por distancia, se calculó la correlación entre los valores de diferenciación genética $\left(\mathrm{F}_{\mathrm{st}}\right)$ y las distancias geográficas para todos los pares de localidades utilizando los procedimientos de permutación de Mantel implementados en el software ARLEQUIN (Mantel 1967). Se realizó un Análisis de Varianza Molecular (AMOVA), para conocer la estructura de la población, organizando las muestras en varios grupos geográficos de acuerdo con propuestas de provincias del POT presentadas por varios autores, las cuales se consideran como hipótesis que pueden explicar la estructura genética de las poblaciones. Las agrupaciones consideradas fueron: (i) Provincia Mexicana (Si, J, M, G, O) vs. Central American gap (C, ES) vs. Provincia Panámica (Pa, Pe) (Hasting 2000); (ii) Unidad Mexicana (Si, J, M, G, O) vs. Unidad Panámica (C, ES, Pa, Pe) (Landini et 
Tabla 1. Medidas de diversidad para Holothuria (Halodeima) inornata. Los valores se presentan considerando las poblaciones de la especie, las unidades Mexicana y Panámica, y todos los individuos de la especie. N, Número de individuos; Nh, Número de haplotipos; $\pi$, Diversidad nucleotídica; H, Diversidad haplotípica.

\begin{tabular}{|c|c|c|c|c|c|c|}
\hline Unidad & Localidad & Código & $\mathbf{N}$ & $\mathrm{Nh}$ & $\Pi$ & $\mathbf{H}$ \\
\hline \multirow[t]{6}{*}{ Mexicana } & & & 103 & 47 & 0.0096 & 0.951 \\
\hline & Sinaloa-Playa Cerritos & $\mathrm{Si}$ & 6 & 4 & 0.008 & 0.8 \\
\hline & Jalisco-Isla Cocinas & $\mathrm{J}$ & 17 & 11 & 0.007 & 0.934 \\
\hline & Michoacán-Caleta de Campos & $\mathrm{M}$ & 21 & 16 & 0.012 & 0.948 \\
\hline & Guerrero-Punta Maldonado & G & 30 & 19 & 0.009 & 0.959 \\
\hline & Oaxaca-Puerto Ángel & $\mathrm{O}$ & 29 & 20 & 0.009 & 0.968 \\
\hline \multirow[t]{5}{*}{ Panámica } & & & 117 & 76 & 0.0146 & 0.973 \\
\hline & Chiapas-Puerto Madero & $\mathrm{C}$ & 30 & 23 & 0.016 & 0.977 \\
\hline & El Salvador-Los Cobanos & ES & 32 & 27 & 0.015 & 0.978 \\
\hline & Panamá-San Carlos & $\mathrm{Pa}$ & 28 & 22 & 0.015 & 0.968 \\
\hline & Perú-Isla Foca & $\mathrm{Pe}$ & 27 & 22 & 0.014 & 0.969 \\
\hline Todos los individuos & & & 220 & 118 & 0.017 & 0.979 \\
\hline Varianza & & & & & 0.0000001 & 0.00001 \\
\hline Error estándar & & & & & 0.00037 & 0.004 \\
\hline
\end{tabular}

al. 2001); (iii) Provincia Mexicana (Si, J, M, G, O, C, ES) vs. Provincia Panámica (Pa, Pe) (Briggs 1974). Por otra parte, se utilizó el programa TCS versión 1.21 (http://darwin.uvigo.es/ software/tcs.html)(Clement et al. 2000) que hace aproximaciones tipo redes con el procedimiento de parsimonia estadística con una confianza de $95 \%$.

Demografía histórica.- Considerando la diferenciación entre las poblaciones, se examinó la demografía histórica por medio de la distribución de mismatch, la cual se realizó en ARLEQUIN. También se realizaron las pruebas estadísticas $\mathrm{D}$ de Tajima y $\mathrm{F}_{\text {s }}$ de Fu (Tajima 1989 y Fu 1997). Para estimar el tiempo aproximado de expansión de las poblaciones de $H$. inornata, se utilizó la fórmula $\mathrm{T}=1 \mathrm{t} / 2 \mathrm{uk}$. Se consideró una tasa de mutación de $1.5 \% / \mathrm{m}$.a. para el gen COI estimado para equinoideos (Lessios et al. 2001).

\section{Resultados}

Diversidad genética.- Se detectaron 118 haplotipos en 220 individuos y las diferencias entre dichos haplotipos fueron debidas a 97 sitios variables en los 453 pb secuenciados (21.41\%) (Números de acceso Genbank KM888322 - KM888544). La diversidad haplotípica, expresada como la probabilidad de encontrar dos haplotipos diferentes en la muestra de 223 individuos, fue de 0.979 . La diversidad nucleotídica, la cual expresa

Tabla 2. Estimaciones de $\mathrm{F}_{\mathrm{st}}$ entre las muestras de Holothuria (Halodeima) inornata basados en ADNmt COI (valores en la diagonal inferior); los valores de $p$ significativos se indican con $\left(^{*}\right)=p<0.05$. Los valores del test exacto de diferenciación poblacional se muestran en la parte diagonal superior. $p<0.05$. Los valores de $p$ significativos se indican con $\left(^{*}\right)$. Localidades: (Si): Sinaloa, Playa Cerritos; (J): Jalisco, Isla Cocinas; (M): Michoacán, Caleta Campos; (G): Guerrero, Punta Maldonado; (O): Oaxaca, Puerto Ángel; (C): Chiapas, Puerto Madero; (ES): El Salvador, Los Cobanos; (Pa): Panamá, San Carlos; (Pe): Perú, Isla Foca.

\begin{tabular}{|c|c|c|c|c|c|}
\hline \multirow[b]{2}{*}{ Localidades } & \multicolumn{5}{|c|}{ Mexicana } \\
\hline & (Si) & (J) & (M) & (G) & (O) \\
\hline (Si) & - & $0.500+0.007$ & $0.150+0.011$ & $0.437+0.015$ & $0.458+0.015$ \\
\hline (J) & -0.009 & - & $0.406+0.011$ & $0.784+0.010$ & $0.707+0.009$ \\
\hline (M) & -0.020 & -0.014 & - & $0.420+0.012$ & $0.729+0.012$ \\
\hline (G) & -0.024 & -0.018 & -0.010 & - & $0.961+0.002$ \\
\hline (O) & -0.035 & -0.009 & -0.011 & -0.019 & - \\
\hline (C) & $0.341^{*}$ & $0.396^{*}$ & $0.336^{*}$ & $0.401^{*}$ & $0.378^{*}$ \\
\hline (ES) & $0.359^{*}$ & $0.416^{*}$ & $0.356^{*}$ & $0.419^{*}$ & $0.394^{*}$ \\
\hline (Pa) & $0.359^{*}$ & $0.414^{*}$ & $0.350^{*}$ & $0.418^{*}$ & $0.395^{*}$ \\
\hline \multirow[t]{2}{*}{$(\mathrm{Pe})$} & $0.434^{*}$ & $0.484^{*}$ & $0.416^{*}$ & $0.481^{*}$ & $0.458^{*}$ \\
\hline & \multicolumn{5}{|c|}{ Panámica } \\
\hline Localidades & (C) & (ES) & (Pa) & $(\mathrm{Pe})$ & \\
\hline (Si) & $0.035+0.003^{*}$ & $0.135+0.014$ & $0.064+0.005$ & $0.108+0.010$ & \\
\hline (J) & $0.012+0.002^{*}$ & $0.004+0.001^{*}$ & $0.002+0.001^{*}$ & $0.003+0.001^{*}$ & \\
\hline (M) & $0.019+0.003^{*}$ & $0.003+0.001^{*}$ & $0.001+0.001^{*}$ & $0.002+0.001^{*}$ & \\
\hline (G) & $0.005+0.003^{*}$ & $0.000+0.000^{*}$ & $0.000+0.000^{*}$ & $0.000+0.000^{*}$ & \\
\hline (O) & $0.003+0.001^{*}$ & $0.004+0.001^{*}$ & $0.000+0.000^{*}$ & $0.000+0.000^{*}$ & \\
\hline (C) & - & $0.122+0.011$ & $0.147+0.014$ & $0.208+0.008$ & \\
\hline (ES) & -0.015 & - & $0.900+0.009$ & $0.964+0.004$ & \\
\hline (Pa) & -0.016 & -0.020 & - & $0.899+0.006$ & \\
\hline (Pe) & -0.013 & -0.019 & -0.017 & - & \\
\hline
\end{tabular}


la probabilidad de encontrar sitios nucleotídicos diferentes entre secuencias de la misma población, fue de $\pi=0.017$ (Tabla 1).

En los análisis por localidades, en general los mayores valores de diversidad haplotípica $(\mathrm{H})$ y nucleotídica $(\pi)$ están en las localidades del sur y los menores en las del norte, que corresponden a las unidades Panámica y Mexicana propuestas por Landini et al. (2001).

Diferenciación poblacional.- Los valores de $\mathrm{F}_{\text {st }}$ fueron altos y significativos al comparar entre las poblaciones de la unidad Mexicana (Si, J, M, G, O) y las de la unidad Panámica (C, ES, $\mathrm{Pa}, \mathrm{Pe})$, observándose nula diferenciación dentro de estos dos grupos. El Test Exacto en la mayoría de los casos corroboró lo encontrado en los estadísticos $\mathrm{F}_{\mathrm{st}}$ (Tabla 2).

Los resultados del análisis de varianza molecular arrojaron que la mayor varianza era explicada significativamente por la diferencia entre los grupos de las poblaciones de la unidad Mexicana y la Panámica (Si-O vs. C-Pe), con 41.95\% ( $\mathrm{p}=0$ ) de varianza total. Esto corrobora lo encontrado en los otros análisis de estructura genética (estadístico $\mathrm{F}_{\mathrm{st}} \mathrm{y}$ test exacto). También se detectó una varianza significativa $(36.09 \%, \mathrm{p}=0.01)$ entre los grupos Provincia Mexicana (Si, J, M, G, O); Central American gap $(\mathrm{C}, \mathrm{ES})$ y Provincia Panámica $(\mathrm{Pa}, \mathrm{Pe})$ propuestas por Hasting (2000). Para la tercera agrupación que consideraba la Provincia Mexicana (Si, J, M, G, O, C, ES) y la Provincia Panámica (Pa, Pe) (Briggs 1974) la varianza explicada fue de $13.71 \%(\mathrm{p}=0.13)$.

La prueba de Mantel obtenida a partir de 10000 permutaciones, arroja una correlación positiva entre la distancia genética y la distancia geográfica lineal; sin embargo, esta correlación no fue alta $(r(A B)=0.508)$. Con base en este resultado, se puede concluir que no existe un patrón de aislamiento por distancia geográfica para $H$. inornata.

Distribución geográfica de los haplotipos de ADNmt.- La red derivada de los datos del gen COI mostró un patrón en el que se observan, de abajo hacia arriba en la figura 2, la mayoría de los haplotipos de Perú, Panamá, El Salvador y Chiapas (unidad Panámica); y hacia arriba los haplotipos de las otras localidades ubicadas hacia el norte Oaxaca, Guerrero, Michoacán, Jalisco y Sinaloa (unidad Mexicana) (Fig. 2)

Los haplotipos más frecuentes: COI-1, COI-2, COI-3 y COI-4, se encontraron en 7.6, 7.2, 6.3 y 5.8\% de los individuos, respectivamente (Fig. 2). El haplotipo más común (COI-1), que por el método se deduce ser el ancestral, fue detectado en todas las localidades muestreadas en la unidad Panámica. El segundo haplotipo (COI-2) más común fue detectado en Chiapas y en todas las localidades de la unidad Mexicana, excepto en Sinaloa. El tercer haplotipo compartido (COI-3) está ampliamente distribuido, encontrándose en todas las localidades de las dos poblaciones, excepto en Chiapas y Sinaloa.

Demografía histórica.- Considerando la diferenciación encontrada entre las poblaciones de las unidades Mexicana y la Panámica, estos análisis se realizaron considerando cada una de estas unidades como una metapoblación panmíctica. La distribución de las diferencias de sitios nucleotídicos entre pares de secuencias mostró una distribución que podría considerarse bimodal en la población Mexicana y multimodal en la población Panámica. Sin embargo, se encontró un buen ajuste al modelo de expansión súbita, en los dos casos la diferencia entre

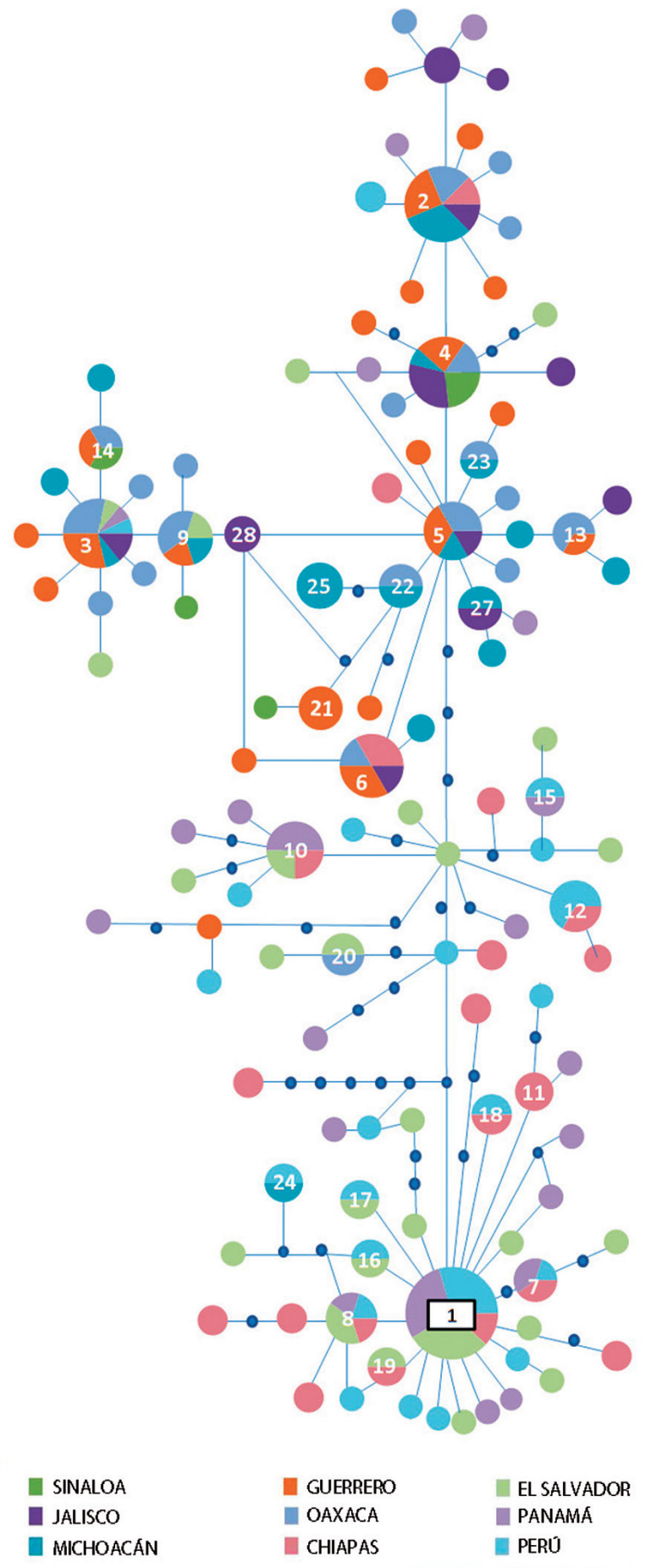

Figura 2. Redes de haplotipos obtenidas con el método de parsimonia estadística con base en las secuencias del gen COI para Holothuria (Halodeima) inornata. El tamaño de los círculos es proporcional al número de individuos que presenta cada haplotipo y las divisiones dentro de cada circulo representan la proporción de individuos de cada localidad, indicadas con colores diferentes. Los haplotipos que solo están en un individuo no tienen número. Cada línea de la red de haplotipos representa un solo cambio mutacional. Los puntos pequeños de color azul indican haplotipos perdidos, probablemente no muestreados o extintos. 
la distribución observada y la esperada bajo este modelo no fue significativa (SSD p>0.05; Fig. 3). Así mismo, las pruebas de neutralidad también indicaron expansión poblacional (Fig. 3).

El tiempo de expansión de las poblaciones de $H$. inornata para la unidad mexicana fue de aproximadamente 344.148 ańos y para la Panámica de 439.555 ańos (Fig. 3).

\section{Discusión}

Con base en el gen COI, Holothuria inornata muestra alta diversidad haplotípica $(\mathrm{Hd}=0.979)$ e intermedia diversidad nucleotídica $(\pi=0.017)$ (Tabla 1$)$, valores que son considerados típicos en invertebrados marinos con tamańos poblacionales grandes (Avise et al. 1984, Watterson 1984). Estos valores son similares a los observados para otras especies de holoturias en otras regiones como el Pacífico Occidental y el Índico (Holothu-
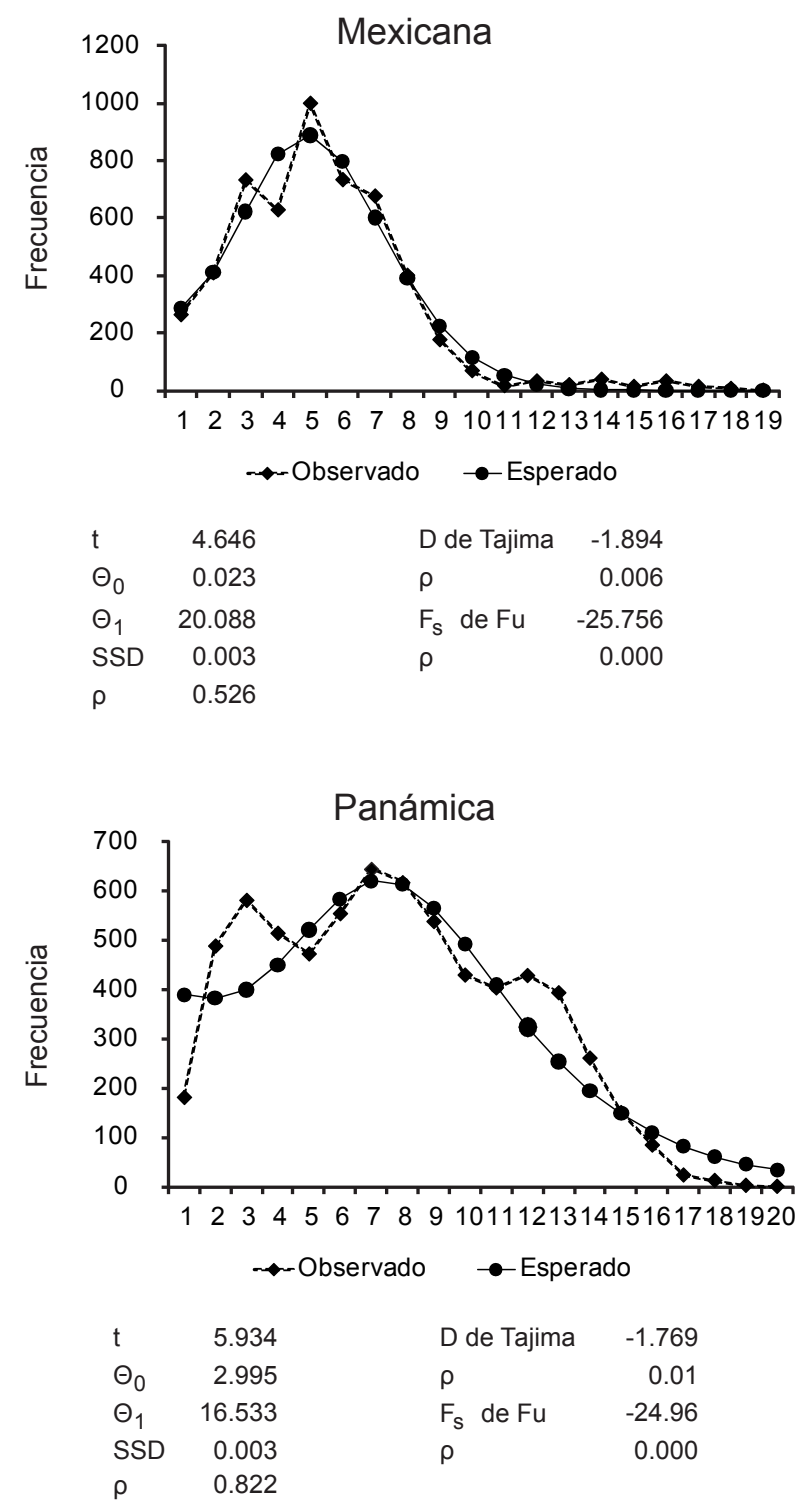

Figura 3. Distribución observada (círculos negros) de las diferencias por parejas y su distribución esperada (círculos vacíos) bajo el modelo de expansión súbita para los grupos definidos para Holothuria (Halodeima) inornata: unidad Mexicana (superior) y unidad Panámica (inferior). Debajo de cada gráfico se incluyen los parámetros de expansión de la población y la prueba de neutralidad. ria atra $\mathrm{Hd}=0.92, \pi=0.009$, Skillings et al. 2011; H. nobilis $\mathrm{Hd}=$ 0.942, $\pi=0.0075$, Uthicke \& Benzie 2003)) y el Atlántico y el Mediterráneo (H. mammata $\mathrm{Hd}=0.92, \pi=0.007$, Borrero-Pérez et al. 2011). Así mismo estos valores son similares a los obtenidos para otras especies de equinodermos (Arbacia lixula $\mathrm{Hd}=0.912$, $\pi=0.0075$, Wangensteen 2013) y otros invertebrados (Nerita albicilla $\mathrm{Hd}=0.989, \pi=0.021$, Crandall et al. 2008; Pachygrapsus crassipes $\mathrm{Hd}=0.923, \pi=0.009$, Cassone \& Boulding 2006). Por otra parte, los valores de diversidad nucleotídica obtenidos en el presente trabajo se encuentra en el intervalo de $0.0005-0.020$, considerado por Stephan y Langley (1992) como valores típicos de la diversidad nucleotídica en una población de acuerdo a los loci y a las especies estudiadas.

Todos los análisis de diferenciación genética entre las localidades muestreadas $\left(\mathrm{F}_{\text {st }}\right.$, test exacto, AMOVA y test de mantel) sugieren que estas podrían dividirse en dos poblaciones, las del norte (Sinaloa, Jalisco, Michoacán, Guerrero y Oaxaca) y las del sur (Chiapas, El Salvador, Panamá y Perú). Esta estructura observada coincide con las unidades Mexicana y Panámica sensu stricto propuestas por Landini et al. (2002). Específicamente en el AMOVA, esta agrupación explicó el mayor porcentaje de varianza con un $41.95 \%$.

Los valores de $\mathrm{F}_{\mathrm{st}}$ obtenidos entre las localidades de las unidades Mexicana y Panámica $(0.336-0.48)$ son altos considerando el intervalo de variación de este estadístico ( 0 hasta 1$)$ y valores mayores a 0.25 indicarían una diferenciación genética muy grande (Wrigth, 1978). Por otro lado, los valores de $\mathrm{F}_{\text {st }}$ dentro de cada una de las unidades son muy bajos, es decir que la diferenciación genética entre las poblaciones de la unidad Mexicana y la Panámica es muy grande, pero el flujo de genes dentro de ellas es muy bajo. Cabe resaltar que el valor de $\mathrm{F}_{\mathrm{st}}$ entre Oaxaca y Chiapas, donde se encuentra el Golfo de Tehuantepec, es alto, del mismo orden de magnitud al encontrado entre localidades distantes, a pesar de la cercanía de estas dos localidades.

La importancia del área del Golfo de Tehuantepec y sus alrededores en la estructura genética de las poblaciones de $H$. inornata en la región del POT se puede observar también cuando se considera el resultado del AMOVA agrupando las localidades de acuerdo a lo propuesto por Hastings (2000), el cual explica un $36 \%$ de la varianza. Según Hastings (2000), las localidades de Chiapas y El Salvador no pertenecerían a ninguna provincia, estarían ubicadas en un tramo de costa denominado Central American Gap, con fondos blandos carentes de substratos rocosos importantes y continuos, este Central American Gap, incluye el área del Golfo de Tehuantepec.

El patrón que se observa en la red de haplotipos obtenida también soporta la estructura poblacional descrita anteriormente. Sin embargo, como es de esperarse se presentan algunos haplotipos, que corresponden al $6 \%$, los cuales son compartidos por poblaciones de ambas unidades. Este patrón puede reflejar el efecto combinado de los eventos históricos y las características oceanográficas actuales que se presentan en el área y que determinan la estructura genética y el flujo genético entre las poblaciones de $H$. inornata. En este sentido la información que podría obtenerse del análisis de estas poblaciones utilizando otros marcadores como los microsatélites, permitiría complementar esta información al describir, por sus características, escenarios más recientes sobre la diversidad y la estructura poblacional. 
El análisis demográfico (distribuciones mismatch, pruebas $\mathrm{D}$ de Tajima y $\mathrm{F}_{\mathrm{s}}$ de $\mathrm{Fu}$ ), considerando a cada población como una unidad panmíctica, indican que ha habido una expansión poblacional (Fig. 3) para ambas. Con base en el cálculo del tiempo aproximado de expansión se podría plantear la hipótesis de que la población de $H$. inornata sufrió una primera expansión en la población Panámica, en la que además se encuentra el haplotipo ancestral, hace aproximadamente 400000 años, y posteriormente una expansión en la población Mexicana hace aproximadamente 300000 ańos (Fig. 3).

Los resultados sugieren que la principal barrera al flujo génico estaría asociada al área del Golfo de Tehuantepec, donde varios factores podrían haber constituido o constituyen actualmente una barrera para el intercambio de larvas y por lo tanto para el flujo de genes. Este golfo está caracterizado por una morfología y oceanografía que incluyen la ausencia de afloramientos rocosos importantes y continuos, la desembocadura de ríos que acarrean sedimentos arenosos desde el continente, la alta actividad biológica por las surgencias eólicas y la direccionalidad hacia el este de la Contra Corriente Ecuatorial. Estos factores podrían impedir el asentamiento larvario, disminuyendo la supervivencia de la especie en el área, lo cual favorecería la diferenciación genética entre las poblaciones de las provincias Mexicana y Panámica.

El conocimiento de la diversidad y a la estructura genética de las poblaciones de $H$. inornata proporcionarían una base científica sólida para implementar políticas de ordenación que permitan el uso sustentable de esta especie como recurso pesquero. En particular la estructura genética de las poblaciones de $H$. inornata nos revela aspectos de su historia evolutiva, información acerca de sus relaciones geográficas y la conectividad entre poblaciones, conocimientos útiles para el planeamiento de estrategias de conservación y restauración, en el caso de ser necesaria.

\section{Agradecimientos}

Agradecemos a todas las personas que colaboraron con la obtención de muestras y su procesamiento, en especial al Dr. Francisco Benítez Villalobos (Universidad del Mar, Oaxaca, México); a Heleni Eunice Cansino Guzmán (Centro de Biociencias, Puerto Chiapas, Chiapas, México); Dr. José Enrique Barraza (Ministerio de Medio Ambiente y Recursos Naturales de El Salvador), Ing. Zedna Ibis Guerra (Laboratorio de Moluscos Bivalvos, Estación de Maricultura del Pacífico, Dirección de Investigación y Desarrollo, Panamá) e Ing. Gustavo N. Collado, Sr. Fabián Valdés y Dr. José Manuel Mazón Suástegui (CIBNOR, La Paz, Baja California Sur, México); a la Dra. Albertina Kameya Kameya (Instituto del Mar del Perú) y a Yuri Hooker (Universidad Peruana Cayetano Heredia). Finalmente a la Dra. Magali Honey Escandón (Posgrado ICML-UNAM) por el apoyo en el desarrollo metodológico del presente trabajo.

Un especial reconocimiento al Dr. Alfredo Laguarda Figueras y al personal del Laboratorio de Sistemática y Ecología de Equinodermos; y al personal del Laboratorio de Genética de Organismos Acuáticos por su importante ayuda.

\section{Literatura citada}

Allauca S. 1990. Presencia de la Corriente Costanera Ecuatoriana. Acta Oceanogr. Pac. 6:10-17.

Alvarado J. J. \& F. A. Solís-Marín. 2013. Echinoderm Research and Diversity in Latin America. Springer. London. 658 pp.
Alvarez L.G., A. Badan-Dangon \& A. Valle. 1989. On coastal currents off Tehuantepec. Estuarine, Coastal and Shelf Science 29 (1): 89-96. doi:10.1016/0272-7714(89)90075-9.

Arndt A. \& M. J. Smith. 1998. Genetic diversity and population structure in two species of sea cucumber: differing patterns according to mode of development. Mol. Ecol. 7:1053-1064.

Avise J. C., J. E. Neigel \& J. Arnod. 1984. Demographic influences on mitochondrial-DNA lineage survivorship in animal populations. Journal of Molecular Evolution. 20:99-105.

Barton E.D., M.F. Lavín, \& A. Trasviña. 2009. Coastal circulation and hydrography in the Gulf of Tehuantepec, México, during winter. Continental Shelf Research 29 (2): 485-500. doi:10.1016/j.csr.2008.12.003.

Benzie J.A.H. 1999. Genetic Structure of Coral Reef Organisms: Ghosts of Dispersal Past. American Zoologist 39 (1): 13145. doi:10.1093/icb/39.1.131.

Borrero-Pérez G. H. 2010. Sistemática y filogeografía de las especies del subgénero Holothuria (Echinodermata: Holothuriidae: Holothuria) de la región Atlanto-Mediterránea. Tesis de doctorado, Universidad de Murcia, Espańa. 228 pp.

Borrero-Pérez G. H., M. González-Wangüemert, C. Marcos \& A. Pérez-Ruzafa. 2011. Phylogeography of the AtlantoMediterranean sea cucumber Holothuria (Holothuria) mammata: the combined effects of historical processes and current oceanographical pattern. Molecular Ecology.1-11.

Brante A., M. Fernández \& F. Viard. 2012. Phylogeography and Biogeography Concordance in the Marine Gastropod Crepipatella Dilatata (Calyptraeidae) along the Southeastern Pacific Coast. Journal of Heredity 103 (5): 630-37. doi:10.1093/ jhered/ess030.

Briggs J. C. 1974. Marine zoogeography. McGraw-Hill. Nueva York.

Briggs J.C. \& B.W. Bowen. 2012. A Realignment of Marine Biogeographic Provinces with Particular Reference to Fish Distributions. Journal of Biogeography 39 (1): 12-30. doi:10.1111/j.1365-2699.2011.02613.x.

Cassone B. J. \& E. G. Boulding. 2006. Genetic structure and phylogeography of the lined shore cral, Pachigrapsus crassipes, along the northeastern and western Pacific coasts. Marine Biology. 149:213-226.

Chang Y., Z. Feng, J. Yu \& J. Ding. 2009. Genetic variability analysis in five populations of sea cucumber Stichopus (Apostichopus) japonicus from China, Russia, South Korea and Japan as revealed by microsatellite markers. Mar. Ecol. 30:455-461.

Clement M., D. Posada \& K. A. Crandall. 2000. TCS: a computer program to estimate gene genealogies. Molecular Ecology. 9:1657-1659.

Coates A., D. McNeill, M. Aubry, W. Berggren \& L. Collins. 2005. An introduction to the geology of the Bocas del Toro archipielago, Panama. Carib J Sci. 41:374-391.

Crandall E., M. A. Frey, R. K. Grosberg \& P. H. Barber. 2008. Contrasting demographic history and phylogeographical patterns in two Indo-Pacific gastropods. Molecular Ecology. $17: 611-626$.

de la Lanza G. 1991. Oceanografía de mares mexicanos. Primera Edición. México. 569 pp.

Excoffier L. \& H.E.L. Lischer. 2010. Arlequin suite ver 3.5: A new series of programs to perform population genetics analyses under Linux and Windows. Molecular Ecology Resources. 10: 564-567. DOI: $10.1111 / j .1755-0998.2010 .02847 . x$

FAO. 2012. Commercially important sea cucumber of the world. FAO Species Catalogue for Fishery Purposes No. 6

Fiedler P.C. 2002. The annual cycle and biological effects of the Costa Rica Dome. Deep Sea Research Part I: Oceanographic Research Papers 49 (2): 321-38. doi:10.1016/S09670637(01)00057-7.

Fu Y. X. 1997. Statistical tests of neutrality of mutations against population growth, hitchhiking and background selection. Genetics. 147:915-925.

Hastings P. 2000. Biogeography of the Tropical Eastern Pacific: distribution and phylogeny of chaenopsid fishes. Zoological Journal of the Linnean Society. 128:319-335.

Hellberg M. E., R. S. Burton, J. E. Neigel \& S. R. Palumbi. 2002. Genetic assessment of connectivity among marine populations. Bulletin of Marine Science. 70(1):273-290 
Honey-Escandón M., A. Laguarda-Figueras \& F. A. Solís-Marín. 2012. Molecular phylogeny of the subgenus Holothuria (Selenkothuria) Deichmann, 1958 (Holothuroidea: Aspidochirotida). Zoological Journal of the Linnean Society. 165:109-120.

Kelly R.P. \& S.R. Palumbi. 2010. Genetic Structure Among 50 Species of the Northeastern Pacific Rocky Intertidal Community. PLoS ONE 5 (1): e8594. doi:10.1371/journal. pone.0008594.

Kessler W. S. 2006. The circulation of the eastern tropical Pacific: a review. Prog. Oceanogr. 69:181-217.

Kinlan B.P. \& S.D. Gaines. 2003. Propagule dispersal in marine and terrestrial environments: a community perspective. Ecology 84 (8): 2007-20. doi:10.1890/01-0622.

Landini W., G. Bianucci, G. Carnevale, L. Ragaini, C. Sorbini, G. Valleri, M. Bisconti, G. Cantalamessa \& C. Di Celma. 2002. Late Pliocene fossils of Ecuador and their role in the development of the Panamic bioprovince after the rising of Central American Isthmus.Can. J. Earth Sci. 39:27-41.

Lessios H.A, B. D. Kessing\& J. S. Pearse. 2001. Population structure and speciation in tropical seas: global phylogeography of the sea urchin Diadema. Evolution.55:955-975

Mantel N. 1967. The detection of disease clustering and a generalized regression approach. Cancer Res. 27:209-220.

Morón O. 2000. Características del ambiente marino frente a la costa peruana. Dirección de Oceanografía Química. Bol. Inst. Mar Perú. 19(1-2):179-204.

Nielsen K.J., S.A. Navarrete. 2004. Mesoscale regulation comes from the bottom-up: intertidal interactions between consumers and upwelling. Ecol. Lett. 7, 31-41. DOI: 10.1046/j.14610248.2003.00542.x

O’Dea A., F. Rodriguez, C. De Gracia \& A. Coates. 2007. La Paleontología marina en el Istmo de Panamá. Canto Rodado. 2:149-179.

Quesada-Alpízar M. \& J. Cortés. 2006. Los exosistemas marinos del Pacífico sur de Costa Rica: estado del conocimiento y perspectivas del manejo. Rev. Biol. Trop. 54 (Suppl. 1):101-145.

Ramos-Ramirez E. 2013. Biología reproductiva de Holothuria (Halodeima) inornata Semper, 1868 en Caleta de Campos, Michoacán, México. Tesis de Licenciatura. Puerto Angel, Oaxaca, México. 53 p.

Raymond M. \& F. Rousset. 1995. An exact test for population diferentiation. Evolution. 49:1280-1283.

Robertson D.R. \& K.L. Cramer. 2009. Shore fishes and biogeographic subdivisions of the tropical Eastern Pacific.Marine Ecology Progress Series, 380, 1-17. doi: 10.3354/meps07925

Roughgarden J. S. Gaines \& H. Possingham. 1988. Recruitment dynamics in complex life cycles. Science (New York, N.Y.) 241 (4872): 1460-66. DOI: 10.1126/science.11538249

Ruiz J. A. 2013. Distribución y abundancia de larvas de langosta roja (Panulirus interruptus) en la costa occidental de la península de Baja California durante 2006-2008. Tesis de Maestría, La Paz, B.C.S., México. 55 pp.

Schultz J. K., K. A. Feldheim, S. H. Gruber, M. V. Ashley, T. M. McGovern \& B. W. Bowen. 2008. Global phylogeography and seascape genetics of the lemon sharks (genus Negaprion). Molecular Ecology. 17:5336-5348.
Skillings D. J., C. E. Bird \& R. J. Toonen. 2011. Gateways to Hawai'i: Genetic Population Structure of the Tropical Sea Cucumber Holothuria atra Journal of Marine Biology. 16 pp.

Solís-Marín F. A., J. Arriaga-Ochoa, A. Laguarda-Figueras, S. Frontana-Uribe \& A. Durán-Gonzales. 2009. Holothuroideos (Echinodermata: Holothuroidea) del Golfo de California. 1 ra. Edición. Instituto de Ciencias del Mar y Limnología. UNAM. CONABIO. 177 pp.

Sotka E.E., J.P. Wares, J.A. Barth, R.K. Grosberg \& S.R. Palumbi. 2004. Strong Genetic Clines and Geographical Variation in Gene Flow in the Rocky Intertidal Barnacle Balanus Glandula. Molecular Ecology 13 (8): 2143-56. doi:10.1111/j.1365294X.2004.02225.x.

Stephan W. \& C. H. Langley. 1992. Evolutionary consequences of DNA mismatch inhibited repair opportunity. Genetics. 132:567-574

Stumpf H.G. 1975. Satellite Detection of Upwelling in the Gulf of Tehuantepec, México. Journal of Physical Oceanography 5 (2): 383-88. doi:10.1175/1520-0485(1975)005<0383:SD OUIT $>2.0 . \mathrm{CO} ; 2$

Tajima F. 1989. The effect of change in population size on DNA polymorphism. Genetics. 123:597-601.

Tellier F., A.P. Meynard, J.A. Correa, S. Faugeron \& M. Valero. 2009. Phylogeographic analyses of the $30^{\circ} \mathrm{S}$ south-east Pacific biogeographic transition zone establish the occurrence of a sharp genetic discontinuity in the kelp Lessonia nigrescens: Vicariance or parapatry?. Molecular Phylogenetics and Evolution 53 (3): 679-93. doi:10.1016/j.ympev.2009.07.030.

Uthicke S. \& J. A. H. Benzie. 2003. Gene flow and population history in high dispersal marine invertebrates: mitochondrial DNA analysis of Holothuria nobilis (Echinodermata: Holothuroidea) populations from the Indo-Pacific. Mol Ecol. 12:2635-2648.

Uthicke S. \& J. A. H. Benzie. 2000. Allozyme electrophoresis indicates high gene flow between populations of Holothuria (Microthele) nobilis (Holothuroidea: Aspidochirotida) on the Great Barrier Reef. Marine Biology. 137:819-825.

Uthicke S., J. Benzie \& E. Ballment. 1998. Genetic structure of fissiparous populations of Holothuria (Halodeima) atra on the Great Barrier Reef. Marine Biology. 132:141-151.

Wangensteen O. S. 2013. Biología y filogeografía del erizo de mar negro Arbacia lixula (Echinoidea: Arbacioida). Tesis de doctorado, Universidad de Barcelona, España. 263 pp.

Watterson G. A. 1984. Allele frequencies after a Bottleneck. Theoretical Population Biology. 26:387-407.

Wright S. 1951. The genetical structure of pupulations.Annals of Eugenics. 15:323-354.

Wright S. 1978. Evolution and the genetics of populations variability within and among natural populations. The University of Chicago Press, Chicago.

Wyrtki K. 1965. Surface currents the Eastern Tropical Pacific Ocean. Interamerican Tropical Tuna Commission Bulletin. 9(5):269-304

Zakas C., J. Binford, S.A. Navarrete \& J.P. Wares. 2009. Restricted gene flow in Chilean barnacles reflects an oceanographic and biogeographic transition zone. Marine Ecology Progress Series 394 (noviembre): 165-77. doi:10.3354/meps08265. 